\title{
Plastic Deformation of InSb Micro-Pillars: A Comparative Study Between Spatially Resolved Laue and Monochromatic $X-R a y$ Micro-Diffraction Maps
}

\author{
Tarik Sadat ${ }^{1, a,{ }^{*}}$, Mariana Verezhak $^{2, b}$, Pierre Godard ${ }^{1, c}$, Pierre Olivier Renault ${ }^{1, d}$, \\ Steven Van Petegem ${ }^{2, \mathrm{e}}$, Vincent Jacques ${ }^{3, \mathrm{f}}$, Ana Diaz ${ }^{2, \mathrm{~g}}$, Daniel Grolimund ${ }^{2, \mathrm{~h}}$, \\ Ludovic Thilly ${ }^{1, i}$ \\ ${ }^{1}$ Institut Pprime, CNRS, Université de Poitiers, SP2MI, Futuroscope Chasseneuil, France \\ ${ }^{2}$ Paul Scherrer Institut, $\mathrm{CH}-5232$ Villigen PSI, Switzerland \\ ${ }^{3}$ Lab. Physique des Solides, CNRS, Université Paris-Sud, Orsay, France \\ atarik.sadat@univ-poitiers.fr, ${ }^{b}$ mariana.verezhak@psi.ch, ${ }^{c}$ pierre.godard@univ-poitiers.fr, \\ d pierre.olivier.renault@univ-poitiers.fr, ${ }^{\mathrm{e}}$ steven.vanpetegem@psi.ch, ${ }^{\mathrm{f}}$ vincent.jacques@u- \\ psud.fr, ${ }^{9} a n a . d i a z @ p s i . c h, ~{ }^{h}$ daniel.grolimund@psi.ch, 'ludovic.thilly@univ-poitiers.fr
}

Keywords: Micro-Pillars, InSb, Laue Microdiffraction, Monochromatic Microdiffraction, Synchrotron Radiation, Plasticity

\begin{abstract}
Indium Antimonide (InSb) single-crystalline micro-pillars were mechanically deformed by uniaxial compression loading-unloading cycles up to the beginning of the plastic regime. After deformation, 2D spatially resolved maps were collected via two X-Ray Diffraction (XRD) techniques: polychromatic micro-Laue and monochromatic micro-diffraction. In both techniques, the integrated diffracted intensity shows strong variations inside the pillar. Moreover, the shift and streaking of one spot in polychromatic XRD as well as the lattice strain and tilt components derived from monochromatic XRD reveal that the plastically deformed area is localized on the top of the pillar, in agreement with scanning electron microscopy images. The two XRD techniques are thus providing correlated but yet complementary information.
\end{abstract}

\section{Introduction}

Indium Antimonide (InSb) and other semiconductors are known to be crystalline materials with brittle behavior at room temperature in the bulk state, but become ductile in the form of microand nano-objects [1-2]. At room temperature, InSb micro-pillars plastically deform by the nucleation of partial dislocations at surfaces: after gliding through the pillar, they escape at opposite surfaces (creating slip traces forming a deformation band) but leave in the crystal parallel Stacking Faults (SFs). This mechanism has been verified by post-mortem Scanning Electron Microscopy (SEM) observations and destructive Transmission Electron Microscopy (TEM) characterization [1-2] but also by non-destructive post-mortem study based on coherent X-Ray diffraction [3]. In the latter case, the profile of coherent diffraction patterns of 202 reflection evolves significantly between pristine and plastically-deformed regions: the splitting and streaking of patterns arise from interferences induced by the presence of SFs that are phasedefects creating phase-shifted regions within the pillar [3]. The maximum intensity of diffraction patterns could be related to the number of SFs present in the illuminated volume, and also to the volume of defected region (i.e. the size of the band containing the SFs). This diffraction technique thus proved to be very interesting as a non-destructive way to detect and characterize the presence of plastic defects in nano-and micro-objects. 
In the present work, the same system (InSb micro-pillar) is used to further assess the capacities of micro-diffraction techniques to characterize the impact of plastic deformation (lattice rotation, residual strain, defects storage, etc.) and in particular compare two techniques, Laue and monochromatic micro-diffraction, via spatially resolved 2D maps that are compared and discussed.

\section{InSb micro-pillars - Mechanical tests}

InSb micro-pillars were fabricated by $\mathrm{Ga}^{+}$Focused Ion Beam (FIB) milling from a bulk $<123>-$ oriented single-crystalline wedge: at all milling steps, the ion beam current and acceleration voltage were kept as low as possible to avoid creation of lattice defects and/or surface amorphisation; for instance, the last milling step was performed at a beam current of $24 \mathrm{pA}$. The resulting geometry is a series of free-standing micro-pillars on top of pedestals. Fig. 1 (a) shows a SEM micrograph of the InSb micro-pillar of interest, with a dimension of $2.5 \times 2.5 \times 7.5 \mu^{3}$. Fig. 1 (d) provides a 3D schematic view of the pillar on its pedestal as well as the relevant crystallographic directions (top view is also schematized, as discussed later). The <123> orientation was chosen to favor "single-slip" deformation during compression, i.e. partial dislocations will nucleate and glide only in a single glide plane, (1-11), resulting in the accumulation of SFs in parallel (1-11) planes [1-3].

(a)

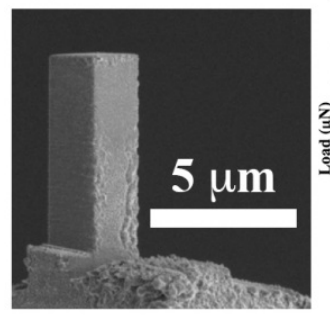

(b)

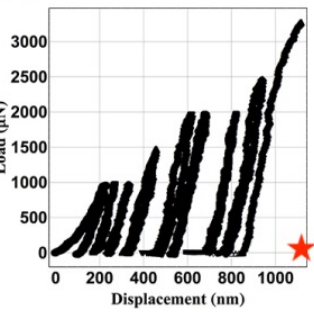

(c)

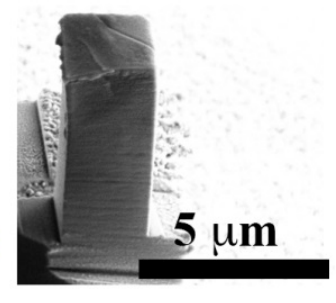

(d)

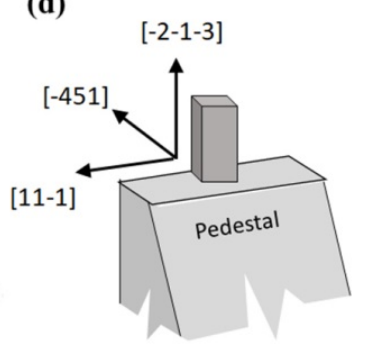

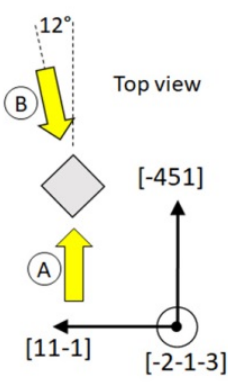

Figure 1: (a) SEM image of studied InSb pillar before deformation. (b) Load versus displacement compression curves applied to the pillar; the (red) star illustrates the condition at which 2D XRD maps were collected. (c) SEM image of the same pillar after deformation. (d) 3D

schematic of pillar on pedestal with crystallographic directions and top view with the two diffraction geometries: "A" refers to the incident beam direction for polychromatic Laue microdiffraction, " $B$ " refers to the incident beam direction for monochromatic micro-diffraction

A custom-designed micro-compression device (MCD), equipped with a diamond flat tip and a standard 1D Triboscope transducer from Hysitron Inc. for force and displacement readout (see [4] for details), was used to in-situ perform uniaxial compression tests combined with a Laue polychromatic micro-diffraction as well as monochromatic micro-diffraction. Here, we focus on the post-mortem study of one specific pillar deformed by a series of loading-unloading cycles until the early plastic regime was attained, as shown by the mechanical curves presented in Fig. 1 (b). After the mechanical test, SEM observations were performed on the pillar, as presented in Fig. 1 (c): the top third of the pillar exhibits permanent bending as a sign of plastic deformation, as well as the presence of two cracks at the very top.

Spatially resolved 2D micro-diffraction maps: comparison between polychromatic and monochromatic cases

After plastic deformation, the pillar was spatially mapped with two different diffraction techniques: polychromatic Laue micro-diffraction and monochromatic micro-diffraction.

Post mortem Laue micro-diffraction mapping was performed at the MicroXAS beamline from the Swiss Light Source (SLS) in Switzerland, with a $1 \times 1 \mu \mathrm{m}^{2}$ pink beam and energy range of [4- 
23] keV. X-Ray Fluorescence (XRF) was used to localize each micro-pillar before and after deformation: XRF maps were obtained by summing the signals from In-L $\alpha, \mathrm{Sb}-\mathrm{L} \alpha$ and $\mathrm{Sb}-\mathrm{L} \beta$ emission yields. A 2D Eiger 4M detector with a pixel size of $75 \times 75 \mu^{2}$ was used to collect Laue patterns in transmission mode. The sample-to-detector distance was fixed at $86.81 \mathrm{~mm}$. $47 \times 24$ patterns were collected from top to bottom of the pillar, with a step size $300 \mathrm{~nm}$. The polychromatic incident beam was parallel to the [-451] direction, corresponding to configuration "A" in top view of Fig. 1 (d). All Laue micro-diffraction data presented here are related to one of the many diffraction spots available in the patterns: the 1-1-1 reflection, as presented in Fig. 2 (a) and (b). A computer code was developed to analyze the data (XRF and XRD) using Python.

Post mortem monochromatic micro-diffraction mapping was performed at the ID01 beamline from the European Synchrotron Radiation Facility (ESRF) in France, with a $8 \mathrm{keV}$ monochromatic beam. A 2D Maxipix detector with a pixel size of $55 \times 55 \mu^{2}$ was used to collect diffraction patterns obtained in reflection mode, with a beam size of about $0.2 \times 0.2 \mu \mathrm{m}^{2}$. The sample-to-detector distance was fixed to $140 \mathrm{~cm}$. 60x60 patterns were collected from top to bottom of the pillar, with a step size of $250 \mathrm{~nm}$. Only the (11-1) reflection was considered (associated to lattice planes that are parallel to pillar and compression axes, see Fig. 1 (d)), as presented in Fig. 2 (c) and (d): constraints from the goniometer and setup imposed that the incident monochromatic beam was at $12^{\circ}$ in opposite direction to [-451], corresponding to configuration "B" in top view of Fig. 1 (d). This will result in mirrored maps obtained from polychromatic and monochromatic micro-diffraction experiments. A rocking curve made of 20 $\phi$-scans was performed to probe the 11-1 Bragg peak in reciprocal space: the $\phi$-scans were done between $18.2^{\circ}$ to $19.15^{\circ}$ with a step size of $0.05^{\circ}$. Homemade programs written in Python and Mathematica were used to analyze collected patterns: in particular, the center of mass for diffracted intensity was calculated as well as the integrated intensity. The conditions of the experiments were such that a horizontal variation of the center of mass of the spot is proportional to a Bragg angle evolution $\Delta \theta$ (in degrees) that can be translated into a lattice spacing evolution $\Delta \mathrm{a}$, or strain $\Delta \mathrm{a} / \mathrm{a}$; a vertical variation of the center of mass of the spot is correlated with a change of the lattice orientation $\Delta \omega$ (in degrees) that can be understood as a tilt (perpendicular to the pillar axis) of the single-crystalline pillar. Note that the other twist component, with an axis aligned with the pillar, could be obtained since rocking curves have been recorded, but is not shown here.
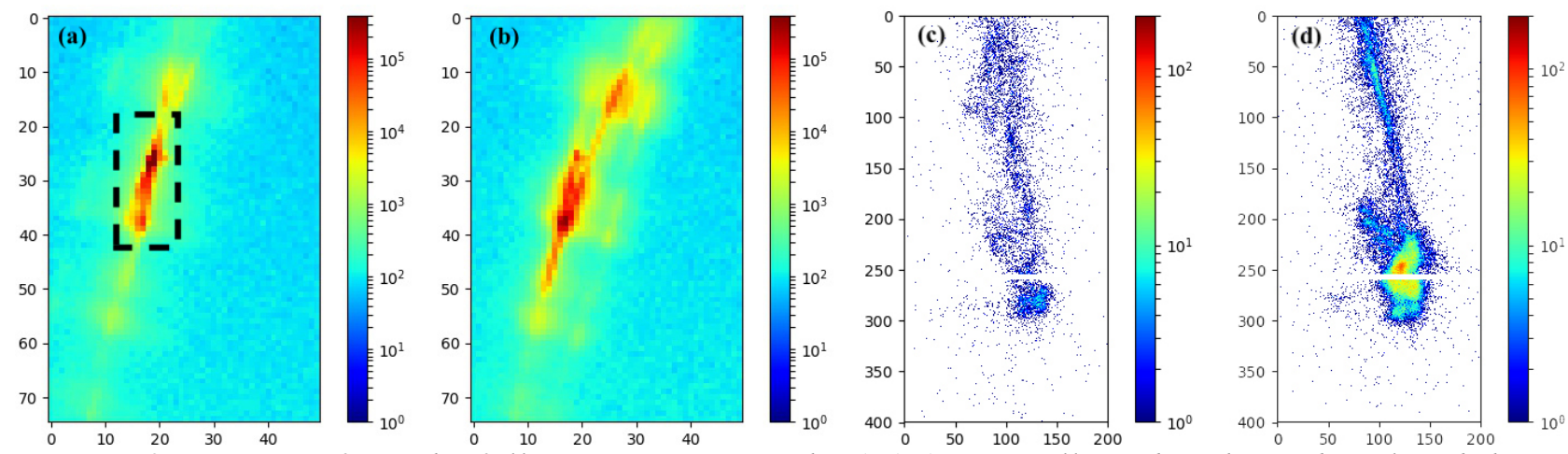

Figure 2: (a) zoom from the full Laue pattern on the 1-1-1 spot collected at the pedestal and (b) at the top of the deformed pillar; the dashed black rectangle is a mask used for analysis. (c) Zoom from the full monochromatic micro-diffraction pattern on 11-1 spot collected at the pedestal and (d) at the top of the pillar. All scales are logarithmic. All axes are in pixels.

Fig. 2 illustrates the shape and intensity of the spots obtained respectively by Laue and monochromatic micro-diffraction at the pedestal (Fig.2 (a) and (c)) and at the top of the pillar (Fig. 2 (b) and (d)), after deformation. It is interesting to observe that in both cases, the shape and 
intensity of the spots are significantly different between the pedestal and the pillar's top, with apparition of peak shift, streaking and splitting that are characteristic of permanent change in lattice strain and orientation, as well as storage of lattice defects: these features are obviously the result of the concentration of plastic deformation at the top of the pillar, as observed in Fig. 1 (c).

Fig. 3 presents the different spatially resolved maps obtained by XRF (Fig. 3 (a)), Laue micro-diffraction (Figure 3 (b)) and monochromatic micro-diffraction (Fig. 3 (c)) on the deformed pillar. The XRF map allows to entirely see the pillar on its pedestal and localize (star symbol) where reference patterns will be taken for the different data analyses. Fig. 3 (b) presents the Laue map of integrated intensity of the 1-1-1 spot (same region as in Fig. 2 (a) and (b)) normalized to the integrated intensity of the same spot at reference position in the pedestal: an unexpected increased normalized integrated intensity is observed in the upper part of deformed pillar, in a region of about $5 \mu \mathrm{m}$ in height. Fig. 3 (c) displays the monochromatic microdiffraction map of integrated intensity of the 11-1 spot normalized to the integrated intensity of the same spot at reference position in the pedestal. The plotted normalized integrated intensity has been obtained from the summation on the 20 rocking curves, or $\phi$-scans, previously described. Again, an increased normalized integrated intensity is observed at top of the pillar; this increase of intensity is more localized than for Laue micro-diffraction, probably because of smaller beam size in monochromatic micro-diffraction. Considering the SEM observations made on the deformed pillar, it seems reasonable to correlate this region of increased diffracted intensity (observed in both maps) to the plastically deformed region at pillar's top.

Fig.4 (a) displays the Laue map of integrated intensity of the 1-1-1 spot, calculated outside the masked region defined in Fig. 2 (a): this region is defined from the pattern at pedestal position, i.e. in a non-deformed region. As a first crude approximation, we consider that the diffracted intensities inside the mask are mainly contributions from the pristine crystal: all intensities outside the mask may then be considered as mainly resulting from plastically deformed regions, inducing shift, streaking and splitting of the 1-1-1 spot. As a result, the obtained map reveals that the most intense region corresponds indeed to the top of the pillar, where plasticity has taken place. Interestingly, this region is more localized than the one revealed previously without any mask (Fig. 3 (b)) and similar to the one revealed by monochromatic micro-diffraction (Fig. 3 (c)). A more in-depth analysis of the Laue spot profile should bring additional information on the origin of observed lattice strain and rotation (incl. lattice defects density) [5]. The monochromatic case, based on a powerful XRD technique [6], has been studied in more details to obtain more quantitative information: Fig. 4 (b) maps the variation in Bragg angle, $\Delta \theta\left(^{\circ}\right)$, deduced from the horizontal shift of center of mass (see above) in patterns collected at a $\phi$ angle equal to $18.2^{\circ}$. Again here, a reference pattern is taken in the pedestal. The Bragg angle variation is then transformed into lattice spacing variation $\Delta \mathrm{a}$, and lattice strain $\Delta \mathrm{a} / \mathrm{a}$, with strain-free InSb lattice being taken as a=6.479 $\AA$ [1-2]. The resulting strain map is presented in Fig. 4 (c): the region with largest residual strain is, without surprise, at the top of the pillar where lattice strain of the order of 0.01 is observed. One should recall that this strain has been calculated from 11-1 spot and is thus associated to the lattice planes parallel to pillar axis (see Fig. 1 (d)): this strain is thus smaller than axial strain by a factor of about $1 / 3$ (Poisson ratio) and of opposite sign. A similar approach has been applied to the vertical shift of the center of mass to obtain a map of lattice orientation variation $\Delta \omega\left(^{\circ}\right)$, or lattice tilt, compared to reference in the pedestal. The map shows that the residual tilt is rather small in the pedestal and in all the bottom part of the pillar: it is only the very top that exhibits relatively large residual tilt values $\left(-0.06^{\circ}\right)$ that are footprints of the plastically bent regions (as observed in SEM) but also the tilted regions between cracks at very top of the pillar. The micro-Laue gives access to a quantification of the tilt. 
Overall, these spatially resolved maps are in good agreement with recent results obtained by Laue micro-diffraction combined with compression to investigate the deformation mechanisms in [001]-oriented single crystal MgO cylindrical pillars: a reduction in diffracted intensity was recorded along the vertical direction, moving from the top towards the bottom of the MgO micro-pillars, and associated to gradual reduction of the strain along the pillars height [7]. In our case, both micro-diffraction techniques maps reveal a localized region at the top of the pillar where the integrated diffracted intensity is higher. This can be explained by the fact that in Laue configuration, a broader part of the pink spectrum is selected in the distorted areas. For the monochromatic case, a similar reasoning can be applied considering the summation over the 20 $\phi$-scans. Moreover, this region is correlated to the volume where residual lattice strain and tilt were induced by plastic deformation
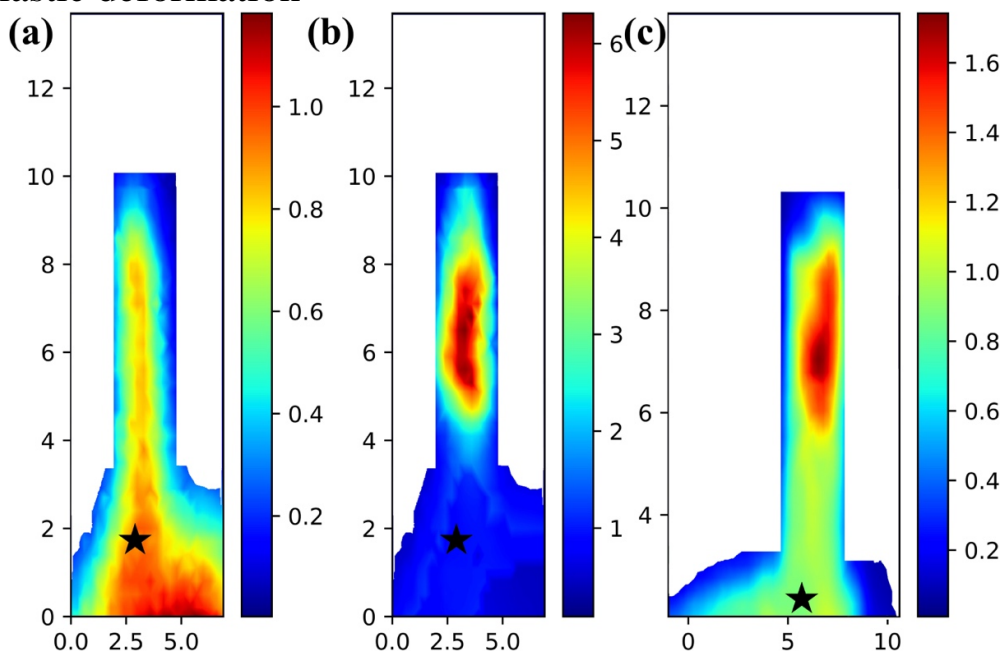

Figure 3: spatially resolved maps from (a) normalized fluorescence of the InSb pillar, (b) normalized integrated intensity of the 1-1-1 spot in Laue micro-diffraction and (c) normalized integrated intensity of the 11-1 spot in monochromatic micro-diffraction. The stars localize the positions for reference patterns. The axes are in $\mu \mathrm{m}$.

(a)

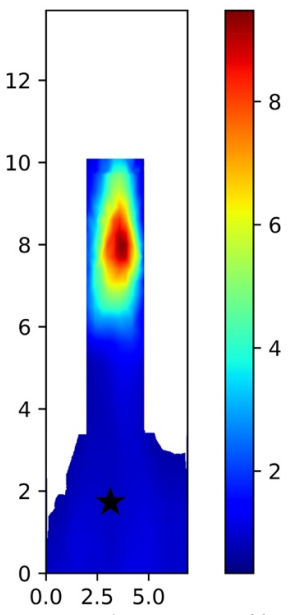

(b)

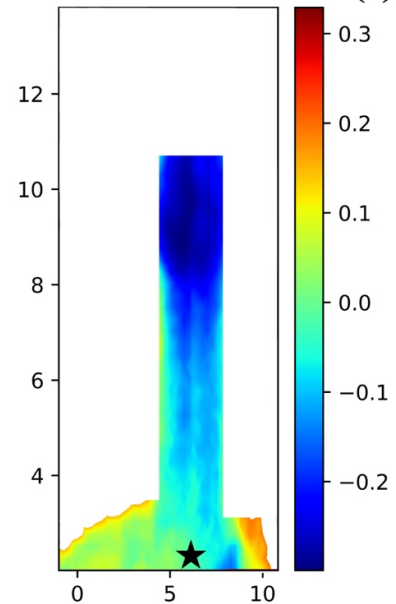

$\Delta \boldsymbol{\theta}\left({ }^{\circ}\right)$

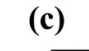

(c)

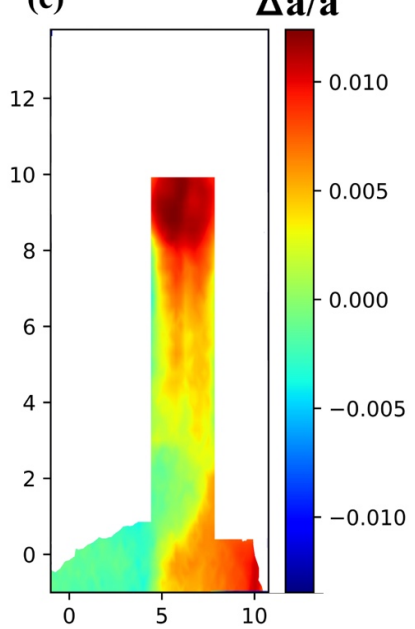

(d)

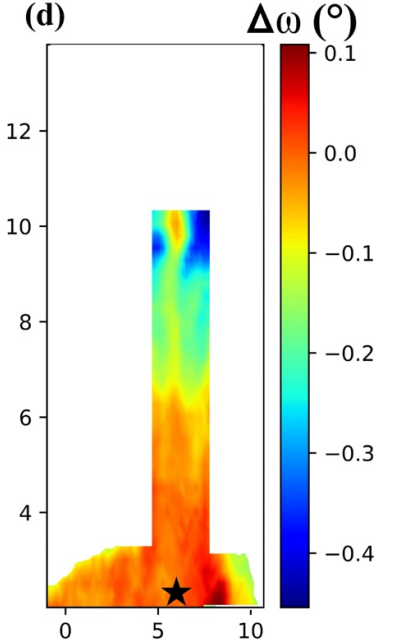

Figure 4: spatially resolved maps of (a) the normalized integrated intensity of the 1-1-1 Laue spot outside a masked region (mask shown on pattern at pedestal in Fig. 2 (a)), (b) the Bragg

angle variation $\Delta \theta\left(^{\circ}\right)$ of the 11-1 reflection in monochromatic micro-diffraction and (c) associated lattice strain $\Delta a / a ;(d)$ the variation $\Delta \omega\left(^{\circ}\right)$ of lattice tilt in the pillar from 11-1 spot monochromatic micro-diffraction. The stars localize the positions for reference patterns. The axes are in $\mu \mathrm{m}$. 


\section{Summary}

We reported here the case of one InSb micro-pillar that was deformed via uniaxial cyclic compression up to the plastic regime. 2D spatially resolved maps obtained from Laue microdiffraction and monochromatic micro-diffraction lead to provide relevant information such as the localization of the plastic activity and increase of strains in a specific region localized at about one third from the top of the pillar and corresponding well to the post-mortem SEM observations. Finally, the two XRD techniques are thus providing correlated but yet complementary information that are useful to better understand the evolution of micro-pillars after mechanical solicitation. This post-mortem benchmarking paves the way for in-situ studies on the same small size objects.

\section{Acknowledgments}

The authors gratefully acknowledge Dario Ferreira Sanchez and Carsten Richter respectively from the MicroXAS (SLS, Switzerland) and ID01 (ESRF, France) beamlines for their support during experiments. Guillaume Amiard is also gratefully acknowledged for the fabrication of the pillars. This project has received funding from the European Union's Horizon 2020 research and innovation program under the Marie Skłodowska-Curie grant agreement No 701647, as well as SNSF grant No 200021L_169753, ANR grant No ANR-16-CE93-0006, and by "Investissement d'Avenir" (LABEX INTERACTIFS, ANR-11-LABX-0017-01) and by Nouvelle Aquitaine Region / European Structural and Investment Funds (ERDF No P-2016-BAFE-94/95).

\section{References}

[1] L. Thilly, R. Ghisleni, C. Swistak, J. Michler, In situ deformation of micro-objects as a tool to uncover the micro-mechanisms of the brittle-to-ductile transition in semiconductors: the case of indium antimonide, Philos. Mag. 92 (2012), 37-41.

https://doi.org/10.1080/14786435.2012.704422

[2] J.M. Wheeler, L. Thilly, A. Morel, A.A. Taylor, A. Montagne, R. Ghisleni, J. Michler, The Plasticity of Indium Antimonide: Insights from Variable Temperature, Strain Rate Jump MicroCompression Testing, Acta Materialia, 106 (2016), 283.

https://doi.org/10.1016/j.actamat.2015.12.036

[3] V.L.R. Jacques, D. Carbone, R. Ghisleni, L. Thilly, Counting dislocations in microcrystals by coherent X-Ray diffraction, Phys. Rev. Lett. 111 (2013), 1-5.

https://doi.org/10.1103/PhysRevLett.111.065503

[4] H. Van Swygenhoven, S. Van Petegem, The use of Laue Microdiffraction to study smallscale plasticity, JOM, 62(12), (2010), 36-43. https://doi.org/10.1007/s11837-010-0178-4

[5] X. Chen, C. Dejoie, T. Jiang, C.-S. Ku, N. Tamura, Quantitative microstructural imaging by scanning Laue X-ray micro- and nanodiffraction, MRS Bull., 41(6), (2016), 445-453. https://doi.org/10.1557/mrs.2016.97

[6] R. Spolenak, W. Ludwig, J. Y. Buffiere, J. Michler, In situ Elastic Strain MeasurementsDiffraction and Spectroscopy, MRS Bull., 35 (5), (2010), 368-374.

https://doi.org/10.1557/mrs2010.569

[7] A. Bhowmik, J. Lee, T.B. Britton, W. Liu, T.-S. Jun, G. Sernicola, M. Karimpour, D.S. Balint, F. Giuliani, Deformation behaviour of [001] oriented MgO using combined in-situ nanoindentation and micro-Laue diffraction, Acta Mater. 145 (2018), 516-531.

https://doi.org/10.1016/j.actamat.2017.12.002 